\title{
Electron-Deficient Group 4 Metal Complexes of Sulfur-bridged Dialkoxide Ligands: Synthesis, Structure and Polymerization Activity Studies
}

Laurent Lavanant, Anca Silvestru, Anne Faucheux, Loic Toupet, Richard F. Jordan, and JeanFrançois Carpentier

Organométalliques et Catalyse, UMR 6509 CNRS-Université de Rennes 1, Institut de Chimie de Rennes, 35042 Rennes Cedex, France

Department of Chemistry, The University of Chicago, 5735 S. Ellis Ave., Chicago, IL 60637, USA

Groupe Matière Condensée et Matériaux, Cristallochimie, UMR 6626 CNRS-Université de Rennes 1, 35042 Rennes Cedex, France

\section{Contents}

I. $\quad$ VT ${ }^{1} \mathrm{H}$ NMR and 2D COSY, HMBC and HMQC experiments for 8

II. 2D COSY, HMBC and HMQC NMR experiments for 15

III. 2D COSY, HMBC and HMQC NMR experiments for 16

IV $\quad{ }^{11} \mathrm{~B}$ NMR and 2D COSY, HMBC and HMQC experiments for $\mathbf{1 7}$

V Representative GPC and DSC analyses of PE samples 
I. 2D (COSY, HMBC and HMQC) and VT ${ }^{1} \mathrm{H}$ NMR experiments for 8

I.1. Selected region (Me resonances) of the VT ${ }^{1} \mathrm{H}$ spectra of 8 (toluene- $d_{8}, 300 \mathrm{MHz}$ )
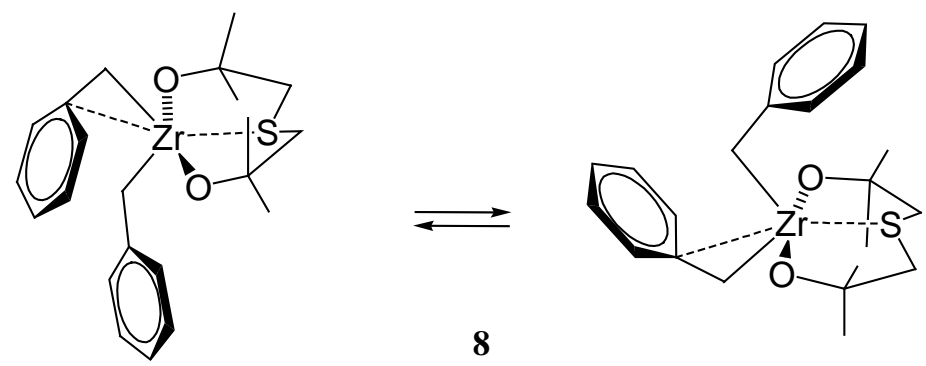

$320 \mathrm{~K}$

$290 \mathrm{~K}$

$280 \mathrm{~K}$

$270 \mathrm{~K}$

$250 \mathrm{~K}$

$240 \mathrm{~K}$

$230 \mathrm{~K}$

$220 \mathrm{~K}$

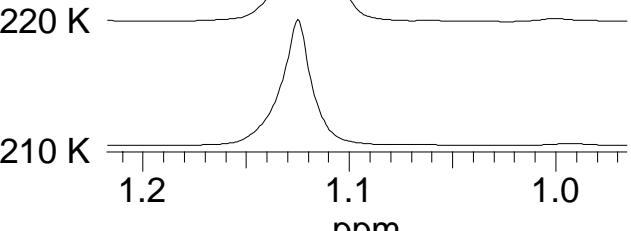

ppm 
I.2. COSY spectrum of 8 (toluene- $d_{8}, 500 \mathrm{MHz}, 240 \mathrm{~K}$ )

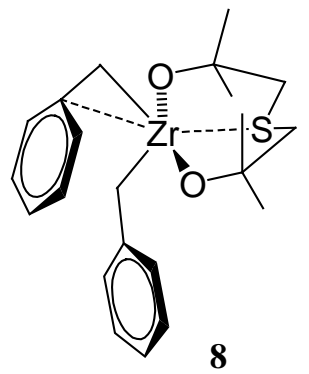

$\operatorname{cog}$ for $\mathrm{B}$

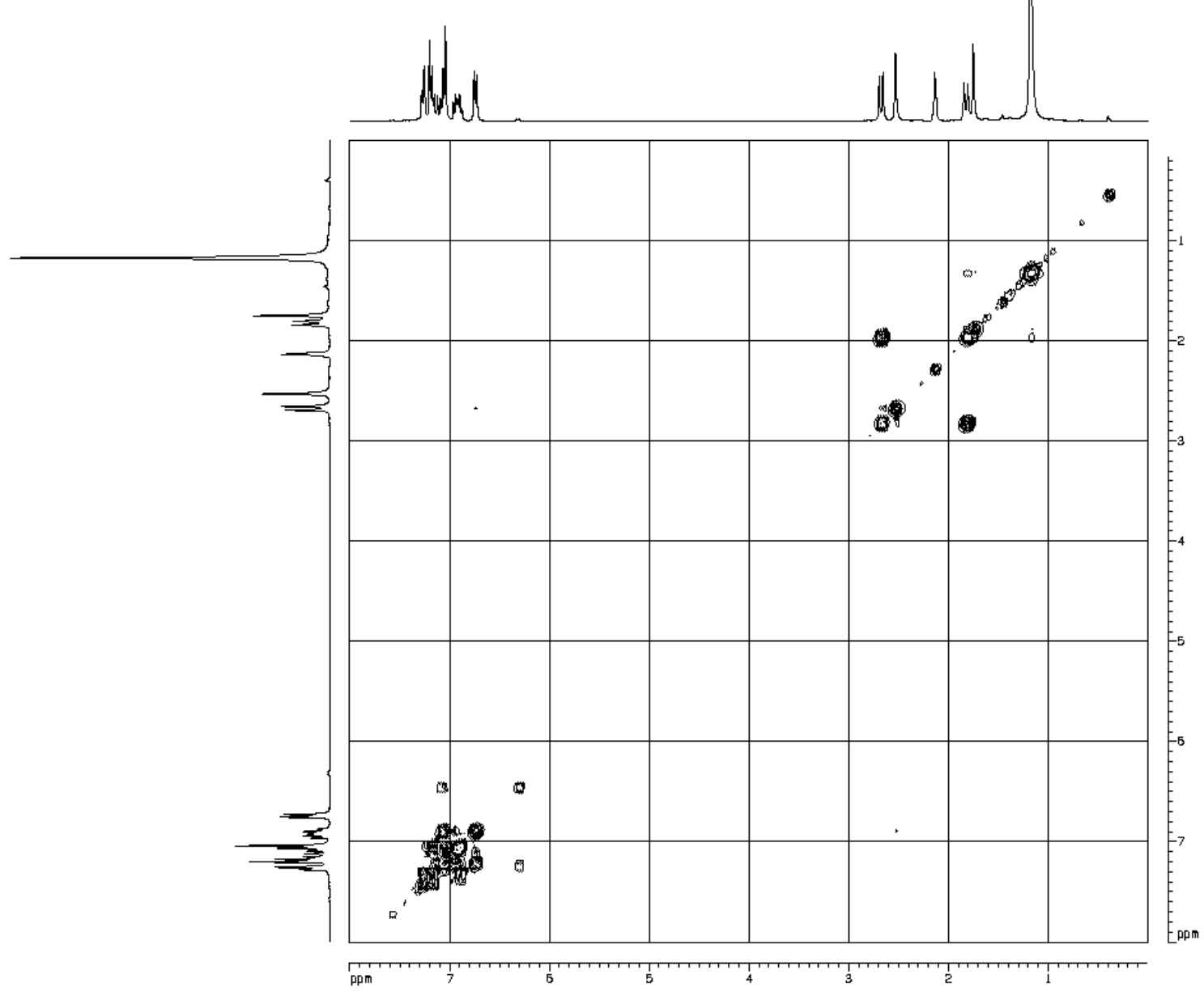


I.3. Selected region of the HMBC spectrum of 8 (toluene- $d_{8}, 500 \mathrm{MHz}$ for ${ }^{1} \mathrm{H}, 240 \mathrm{~K}$ )
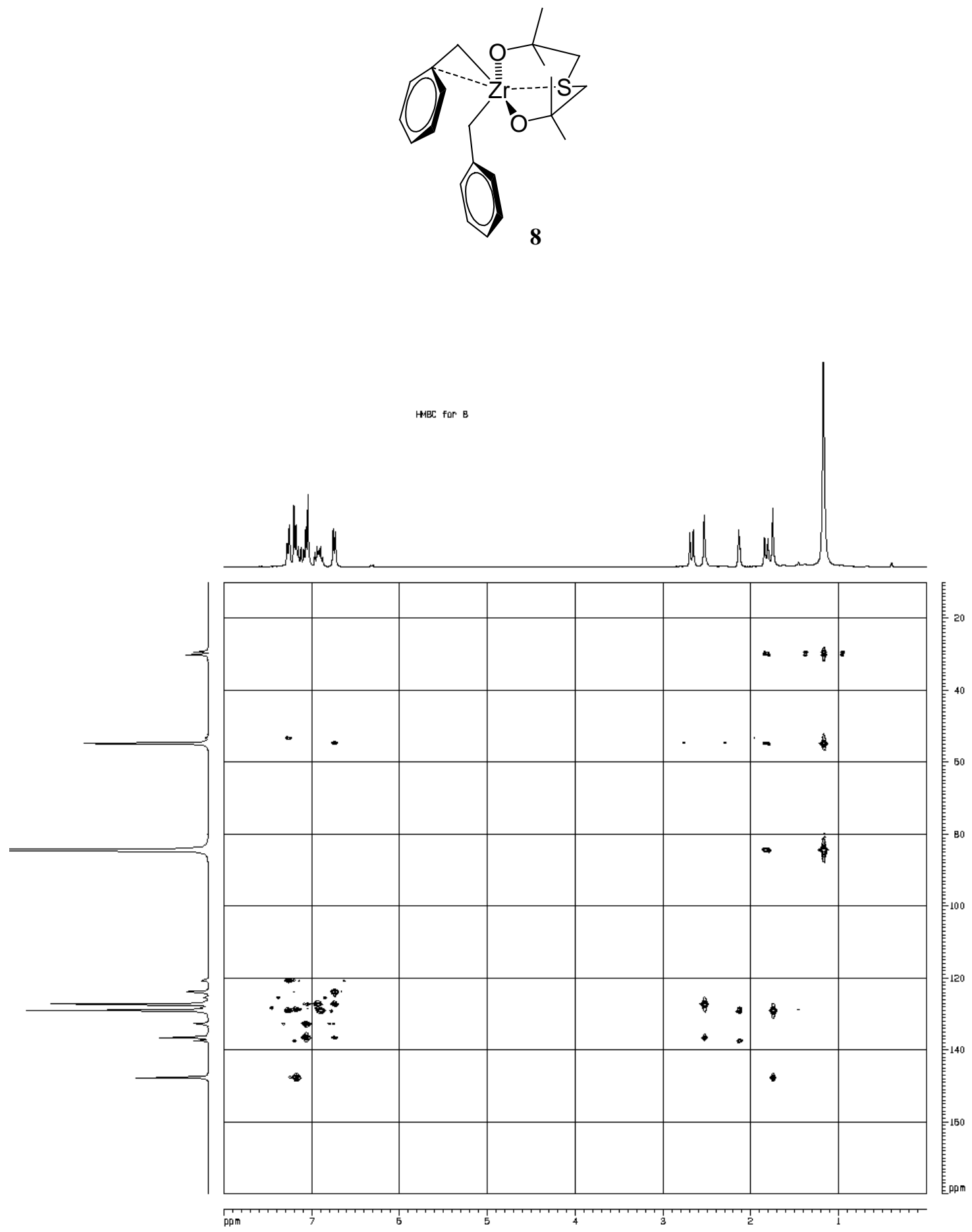
I.4. Selected region of the HMQC spectrum of 8 (toluene- $d_{8}, 500 \mathrm{MHz}$ for ${ }^{1} \mathrm{H}, 240 \mathrm{~K}$ )
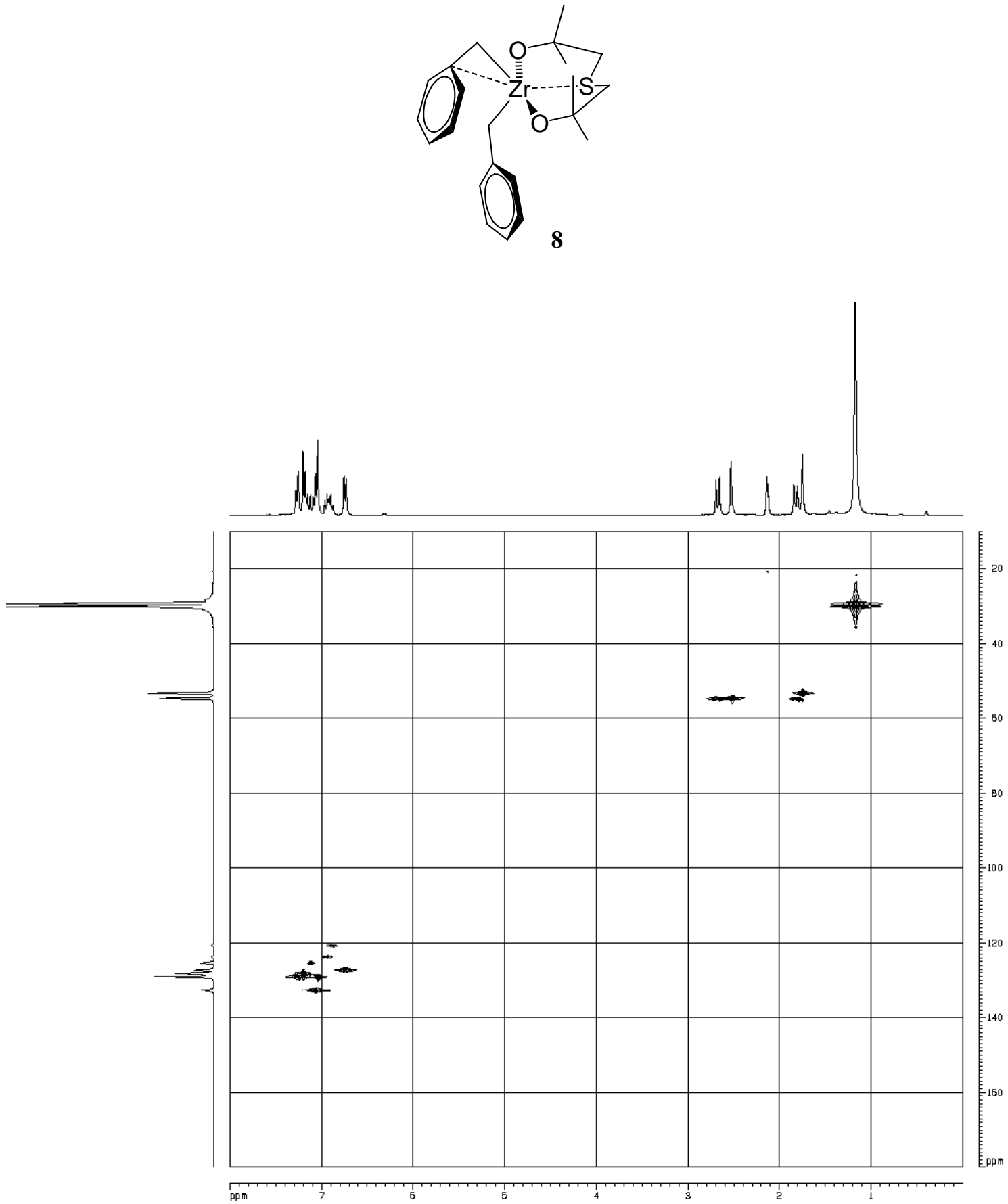
II. 2D COSY, HMBC and HMQC NMR experiments for $\mathbf{1 5}$

II.1. Selected region of the COSY spectrum of $15\left(\mathrm{CD}_{2} \mathrm{Cl}_{2}, 500 \mathrm{MHz}, 240 \mathrm{~K}\right)$
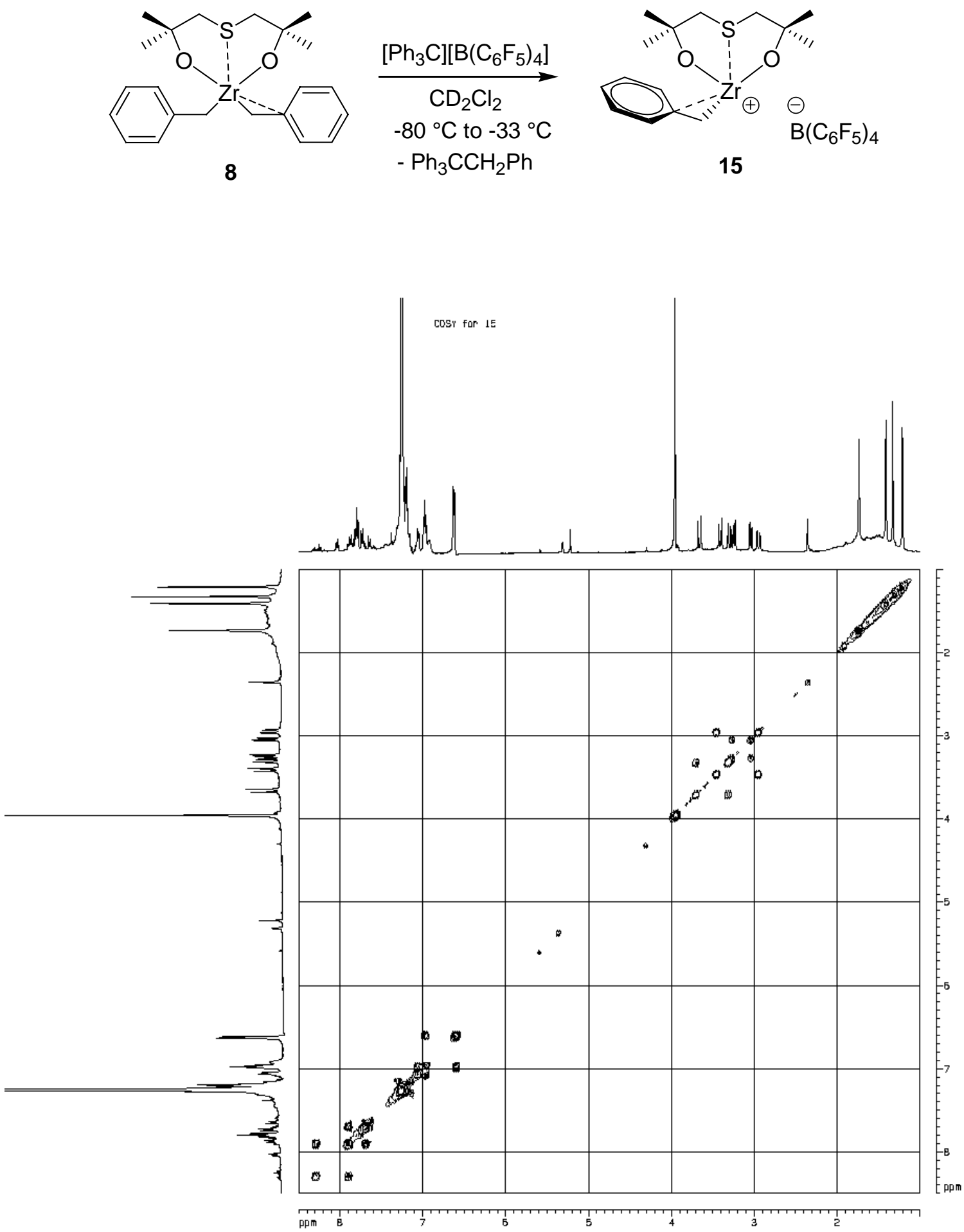
II.2. Selected regions of the $\mathrm{HMBC}$ spectrum of $\mathbf{1 5}\left(\mathrm{CD}_{2} \mathrm{Cl}_{2}, 500 \mathrm{MHz}\right.$ for $\left.{ }^{1} \mathrm{H}, 240 \mathrm{~K}\right)$
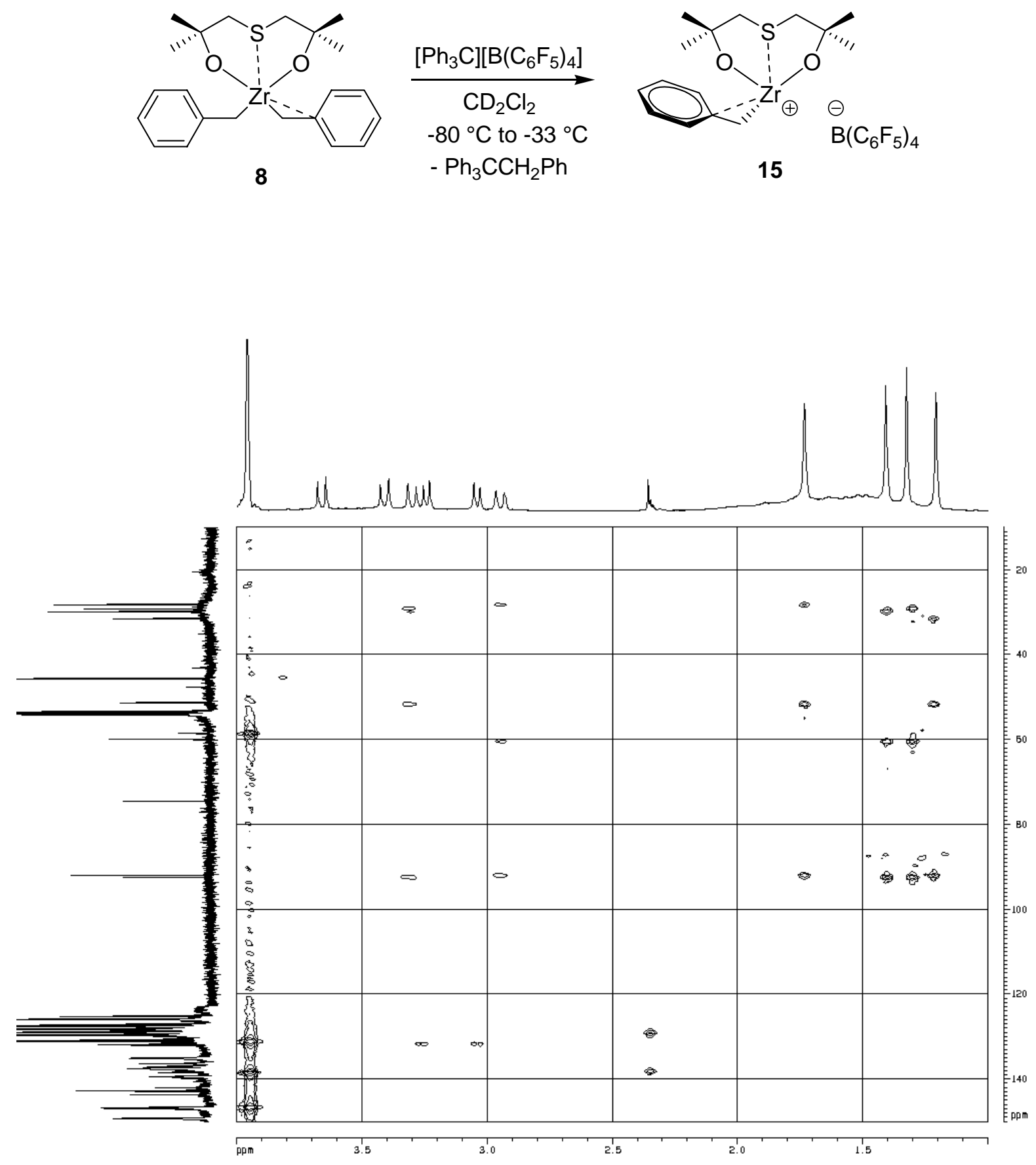
II.2. Selected regions of the HMBC spectrum of $\mathbf{1 5}\left(\mathrm{CD}_{2} \mathrm{Cl}_{2}, 500 \mathrm{MHz}\right.$ for $\left.{ }^{1} \mathrm{H}, 240 \mathrm{~K}\right)$ (continued)

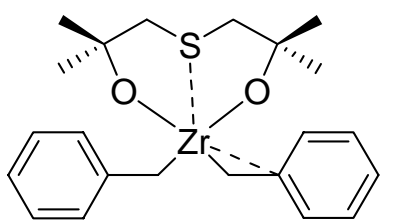

8

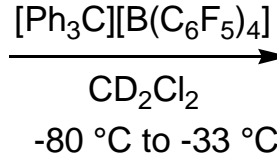

- $\mathrm{Ph}_{3} \mathrm{CCH}_{2} \mathrm{Ph}$

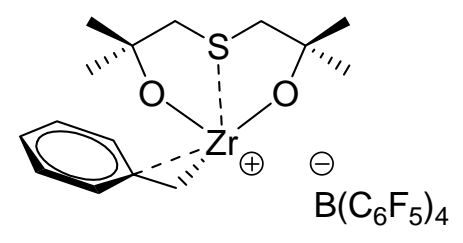

15

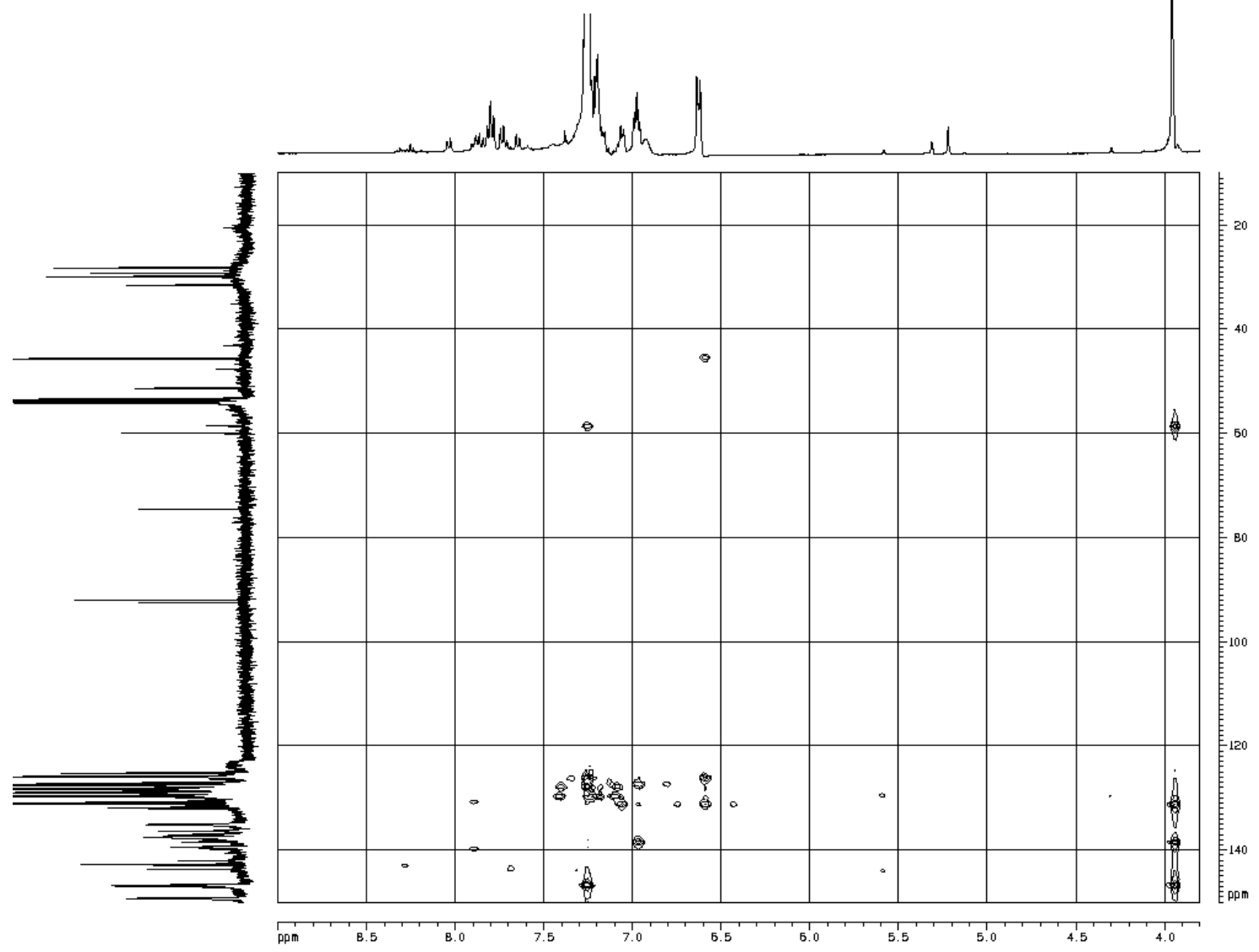


II.3. Selected region of the HMQC spectrum of $\mathbf{1 5}\left(\mathrm{CD}_{2} \mathrm{Cl}_{2}, 500 \mathrm{MHz}\right.$ for $\left.{ }^{1} \mathrm{H}, 240 \mathrm{~K}\right)$
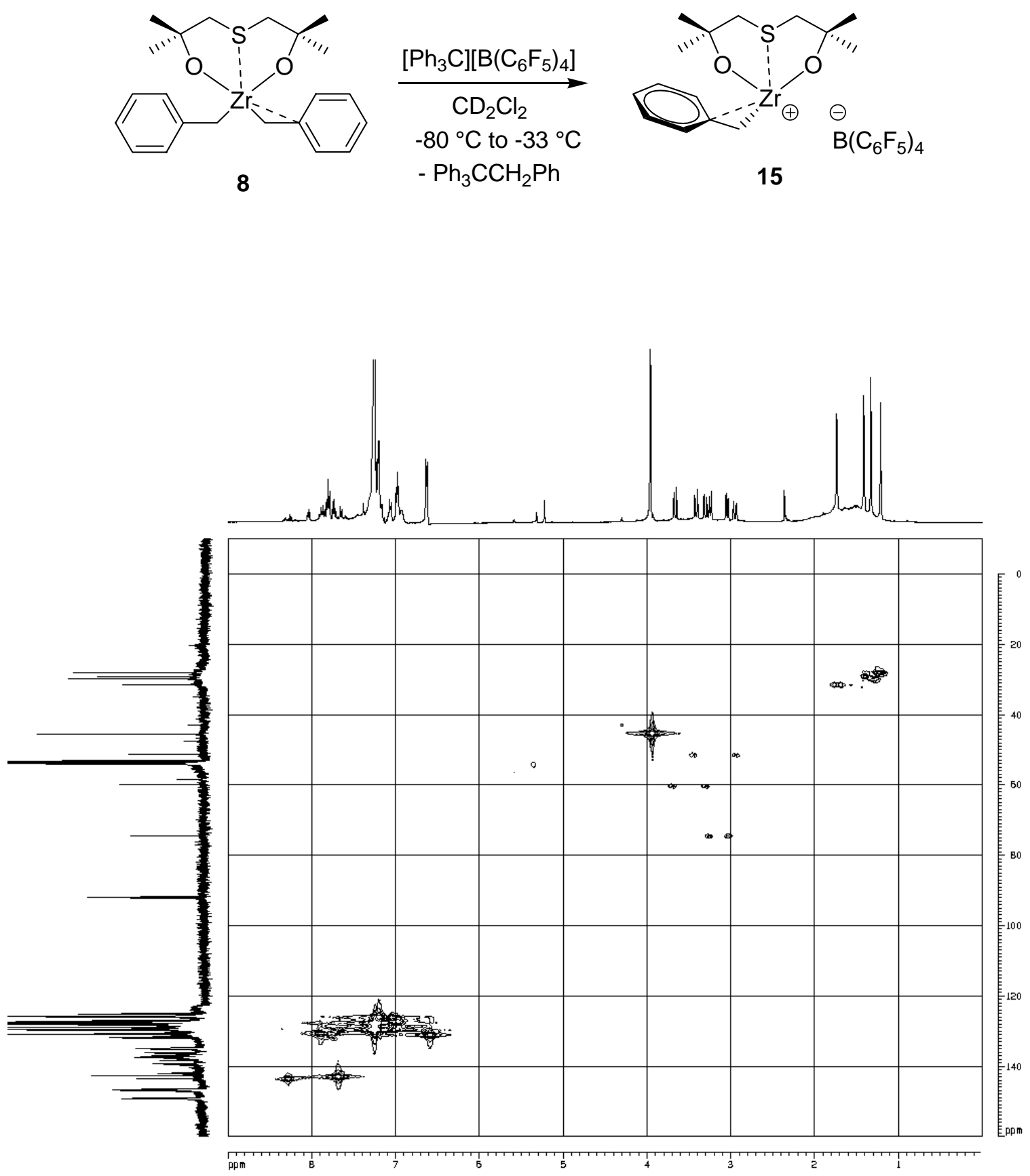
III. 2D COSY, HMBC and HMQC NMR experiments for $\mathbf{1 6}$

III.1. Selected region of the COSY spectrum of 16 (toluene- $d_{8}, 300 \mathrm{MHz}, 240 \mathrm{~K}$ )

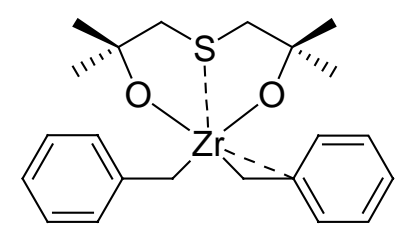

8

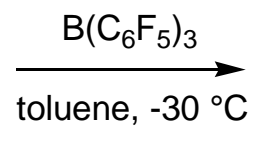

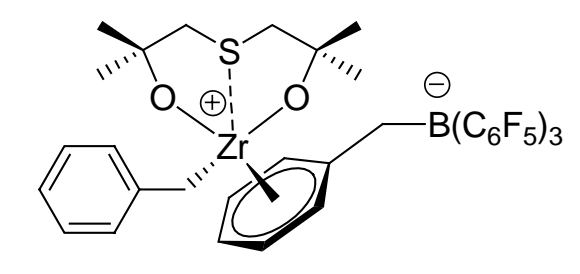

16

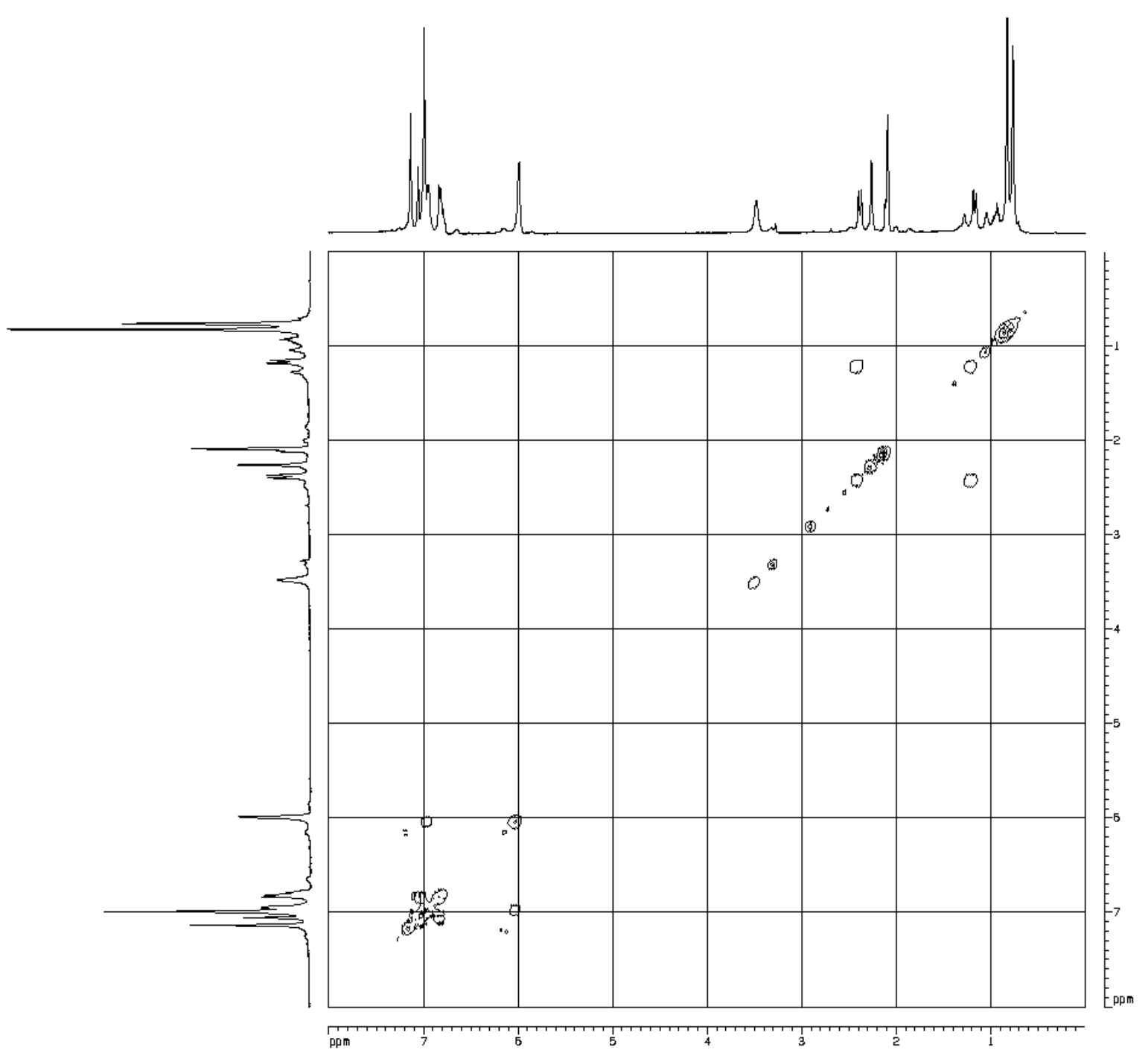


III.2. Selected region of the HMBC spectrum of $\mathbf{1 6}$ (toluene- $d_{8}, 400 \mathrm{MHz}$ for ${ }^{1} \mathrm{H}, 240 \mathrm{~K}$ )

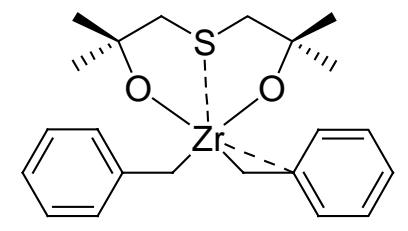

8 $\underset{\text { toluene, }-30^{\circ} \mathrm{C}}{\stackrel{\mathrm{B}\left(\mathrm{C}_{6} \mathrm{~F}_{5}\right)_{3}}{\longrightarrow}}$

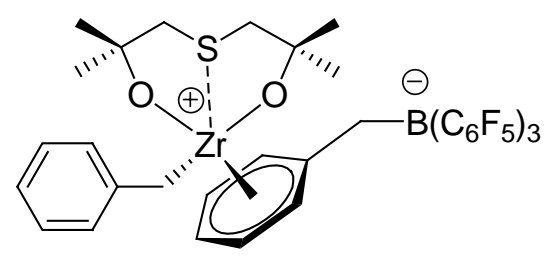

16

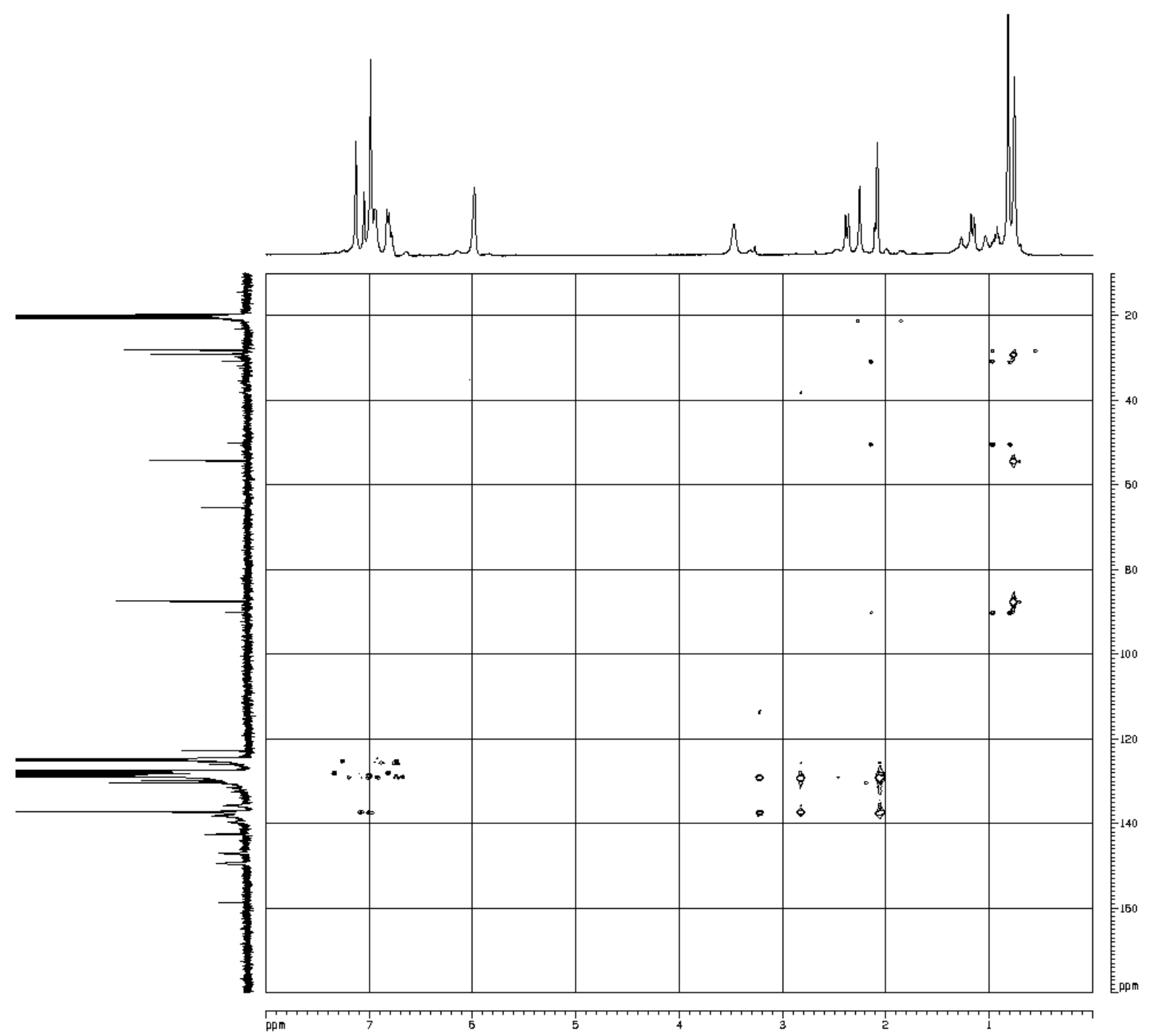


III.3. Selected region of the HMQC spectrum of $\mathbf{1 6}$ (toluene- $d_{8}, 400 \mathrm{MHz}$ for ${ }^{1} \mathrm{H}, 240 \mathrm{~K}$ )

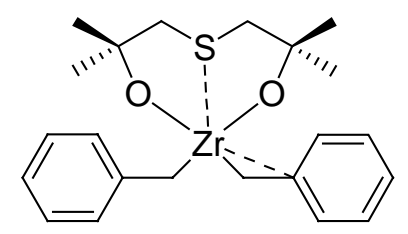

8 $\underset{\text { toluene, }-30{ }^{\circ} \mathrm{C}}{\stackrel{\mathrm{B}\left(\mathrm{C}_{6} \mathrm{~F}_{5}\right)_{3}}{\longrightarrow}}$

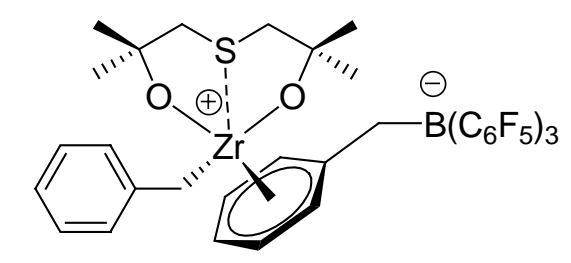

16

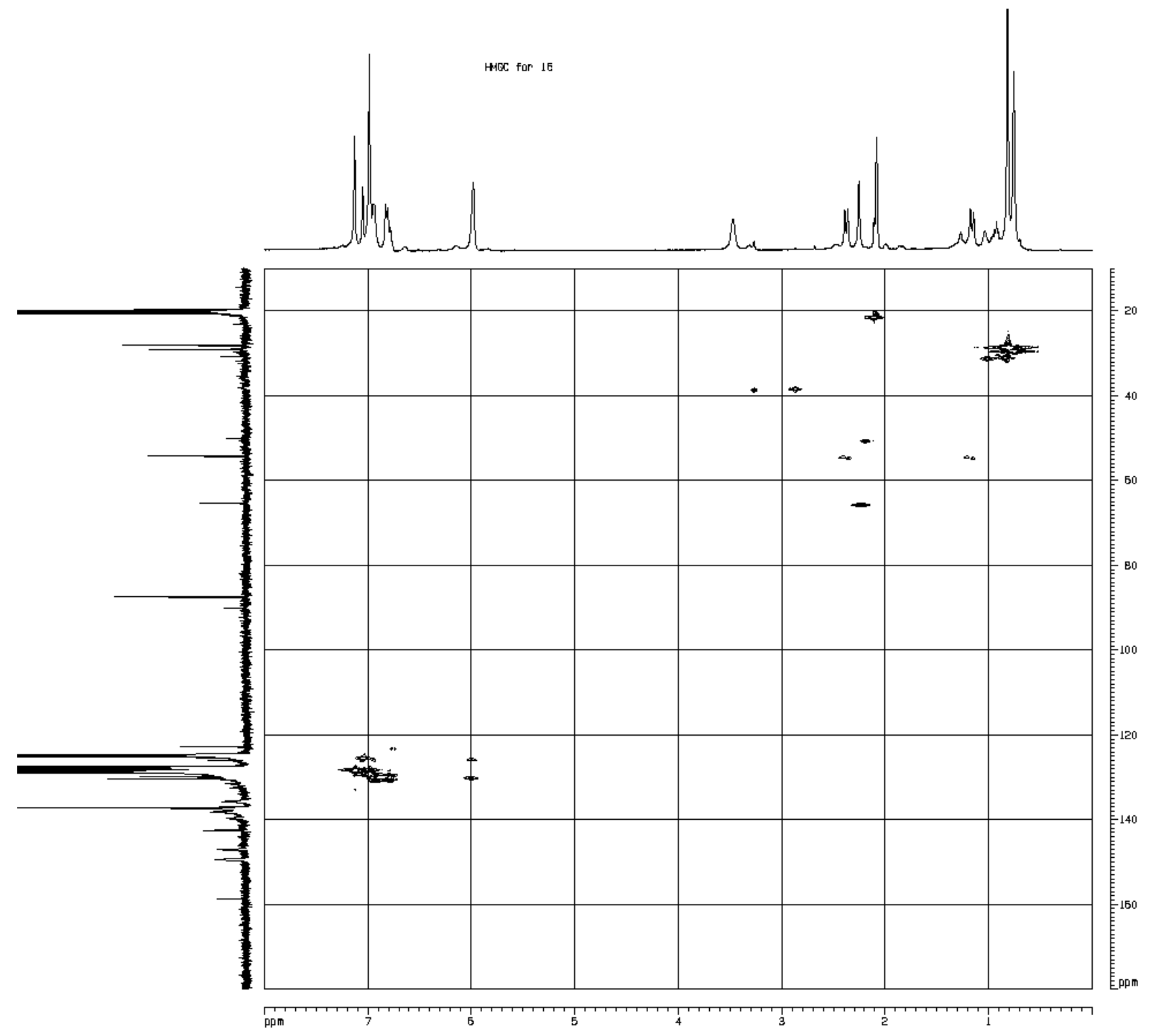


IV. ${ }^{11}$ B NMR and 2D COSY, HMBC and HMQC experiments for $\mathbf{1 7}$

IV.1. ${ }^{11} \mathrm{~B}$ NMR monitoring for the conversion of $\mathbf{1 6}$ to $\mathbf{1 7}$ (toluene- $d_{8}, 128 \mathrm{MHz}, 290 \mathrm{~K}$ )

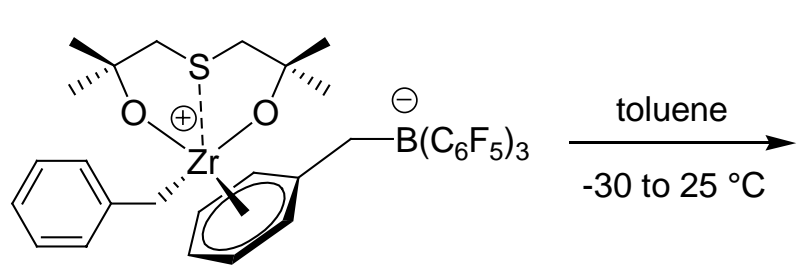

16

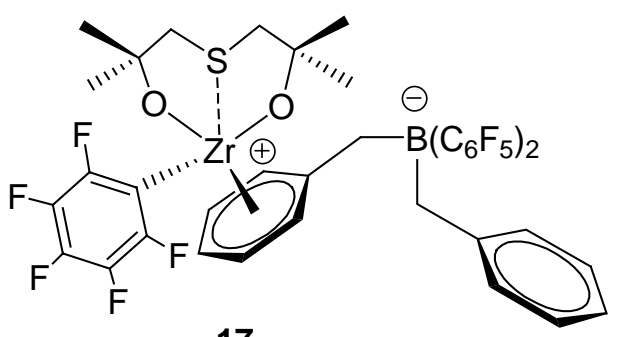

17

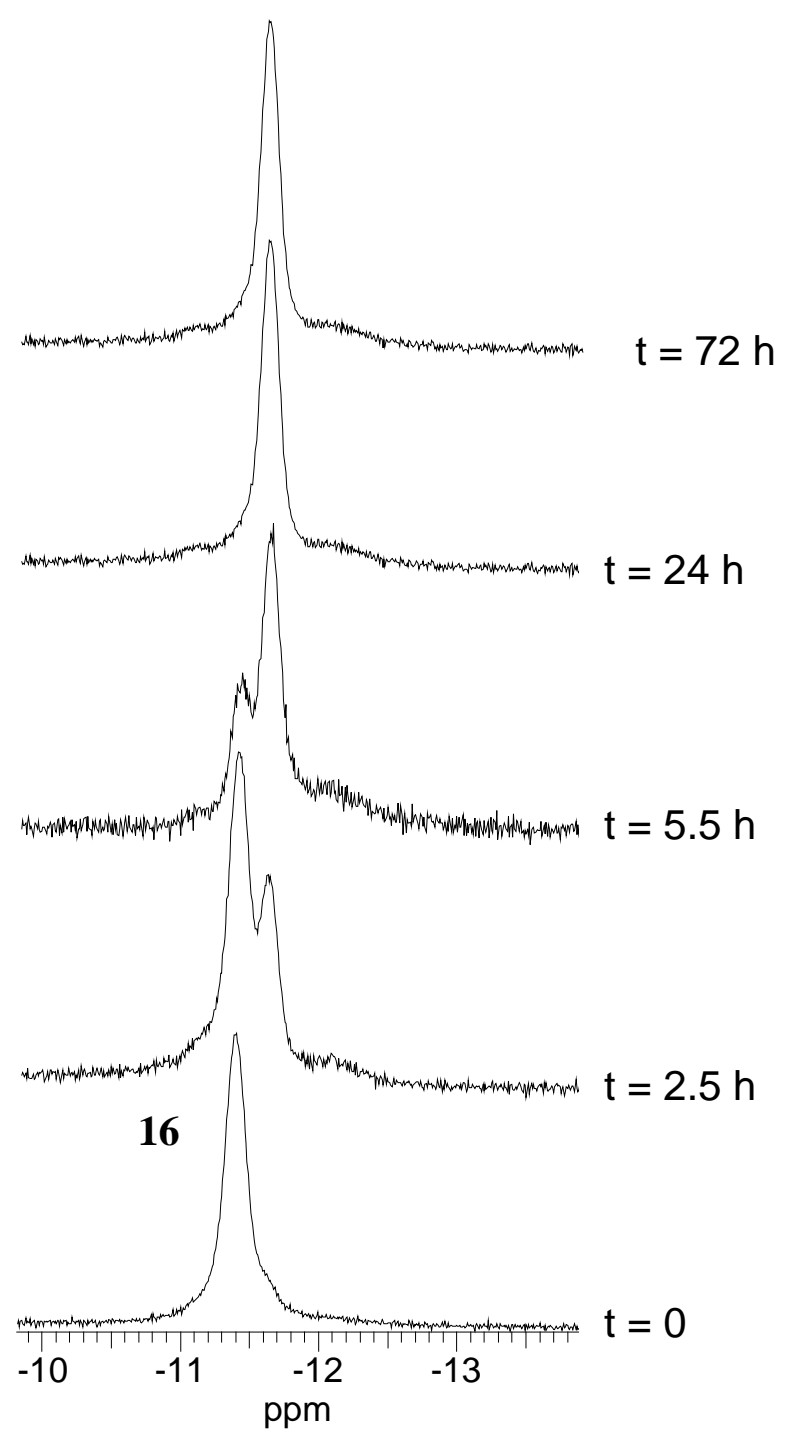


IV.2. Selected region of the COSY spectrum of 17 (toluene- $d_{8}, 400 \mathrm{MHz}, 290 \mathrm{~K}$ )
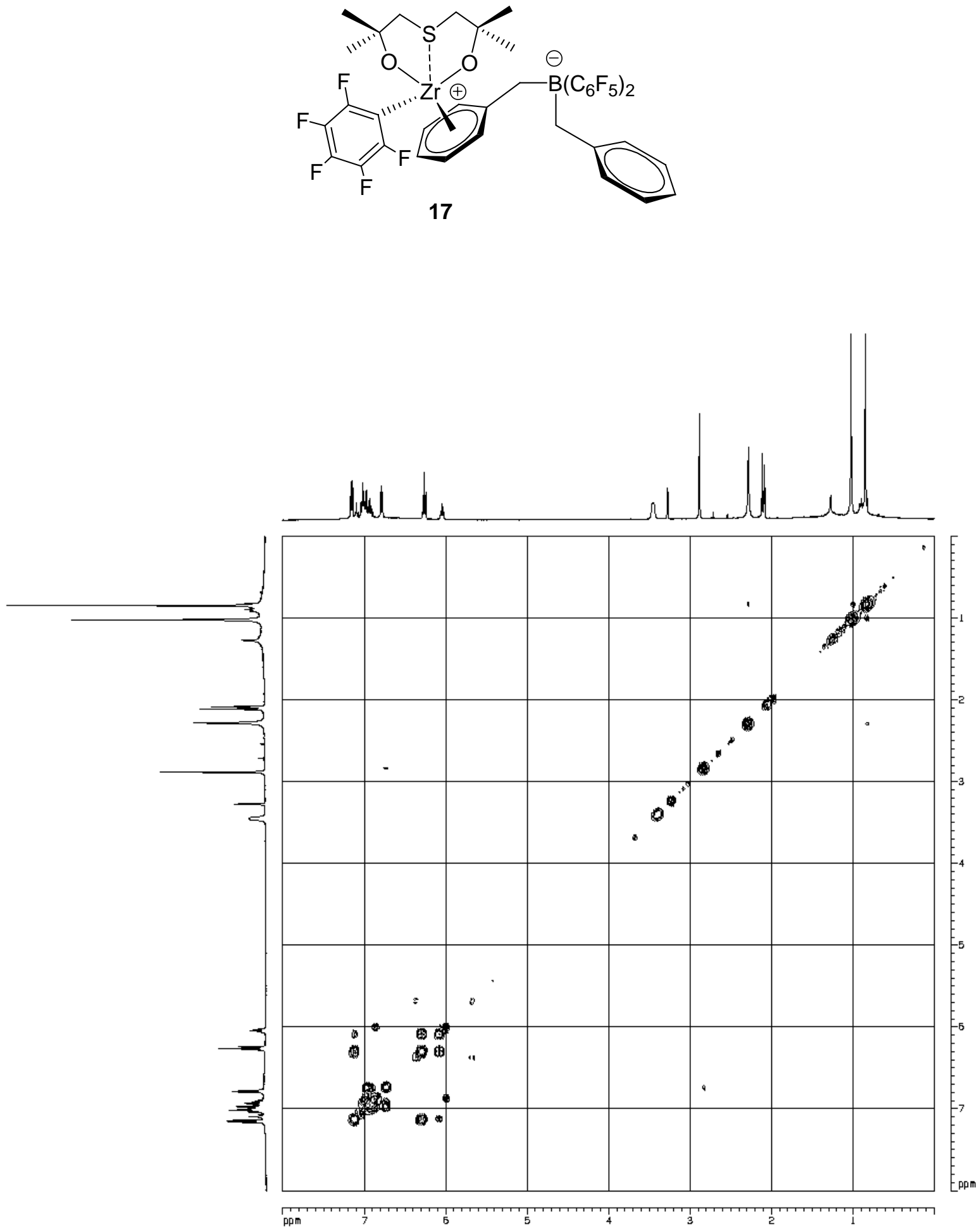
IV.3. Selected region of the HMBC spectrum of $\mathbf{1 7}$ (toluene- $d_{8}, 400 \mathrm{MHz}$ for ${ }^{1} \mathrm{H}, 290 \mathrm{~K}$ )

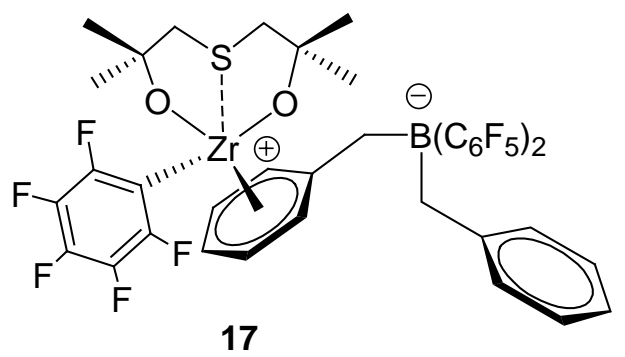

Heder for 17

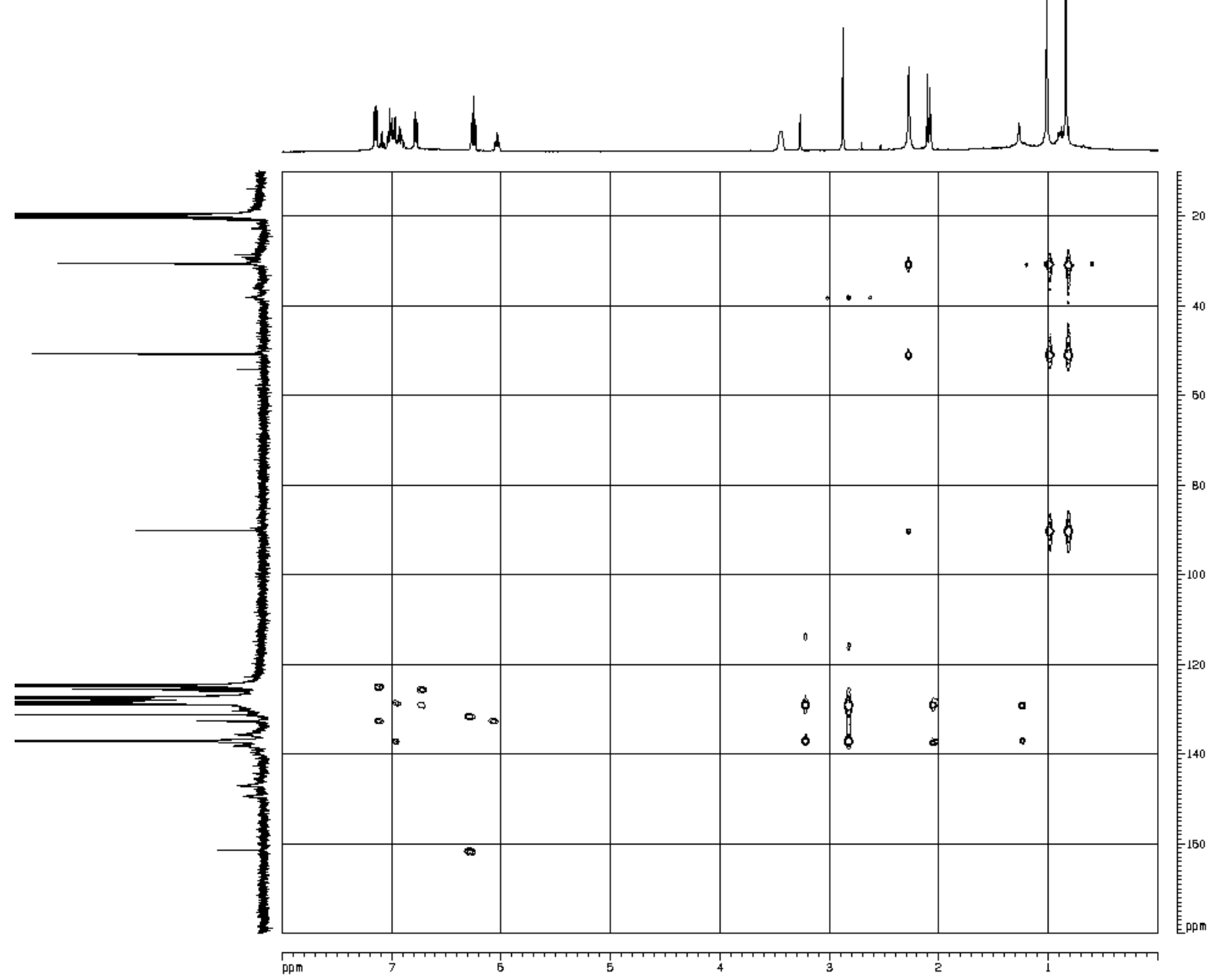


IV .4. Selected region of the HMQC spectrum of 17 (toluene- $d_{8}, 400 \mathrm{MHz}, 290 \mathrm{~K}$ )
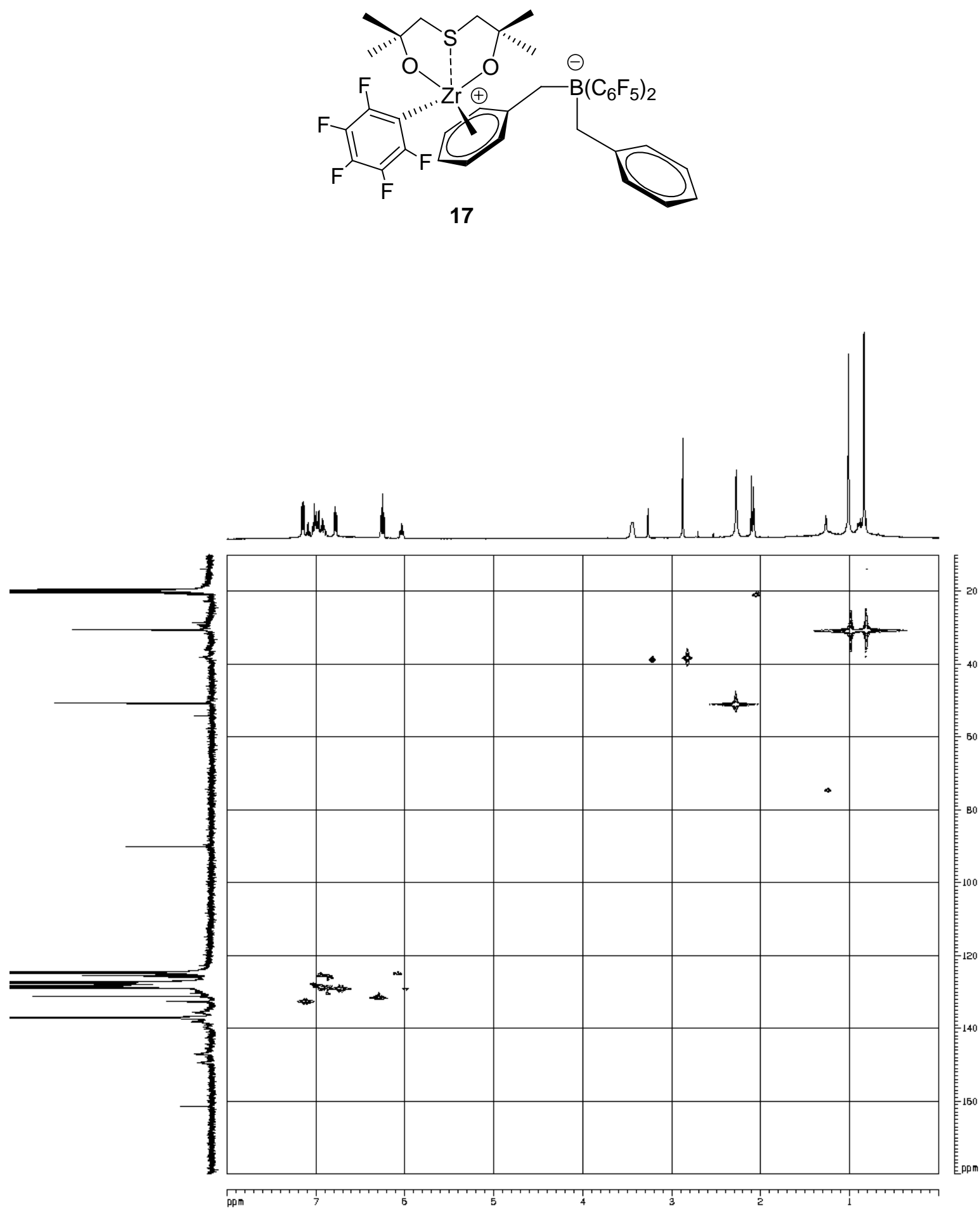

16 
V.1. GPC of PE sample, Table 5, entry 1

Polymer Laboratories

PL Caliber GPC Software

Unknown 76. HOO

$>$ PE

Concentration :

Injection Volume :

Solvent

Column Set

Method 1

Comments :

Calibration Using

Calibration Limits :

Flow Rate Marker :

Narrow Standards

14.08

$\begin{array}{lll}\text { Broad Peak Start : } & 12.88 & \text { End : } 24.73 \quad \text { Mins }\end{array}$

$\begin{array}{lll}\text { Broad Peak Start : } & 12.88 & \text { End : } 24.73 \quad \text { Mins }\end{array}$

\section{9:22 Wed Jun 022004}

Acquired : $\quad$ 08:59 Wed Jun 022004

Operator Richard Jordan

Detector

Temperature :

Flow Rate : 1.000

Standards
Curve Used : 3rd Order Polynomial

Last Calibrated : $\quad$ Wed Jun 02 09:16:52 2004

in Standards at : $\quad 38.42 \quad$ Mins

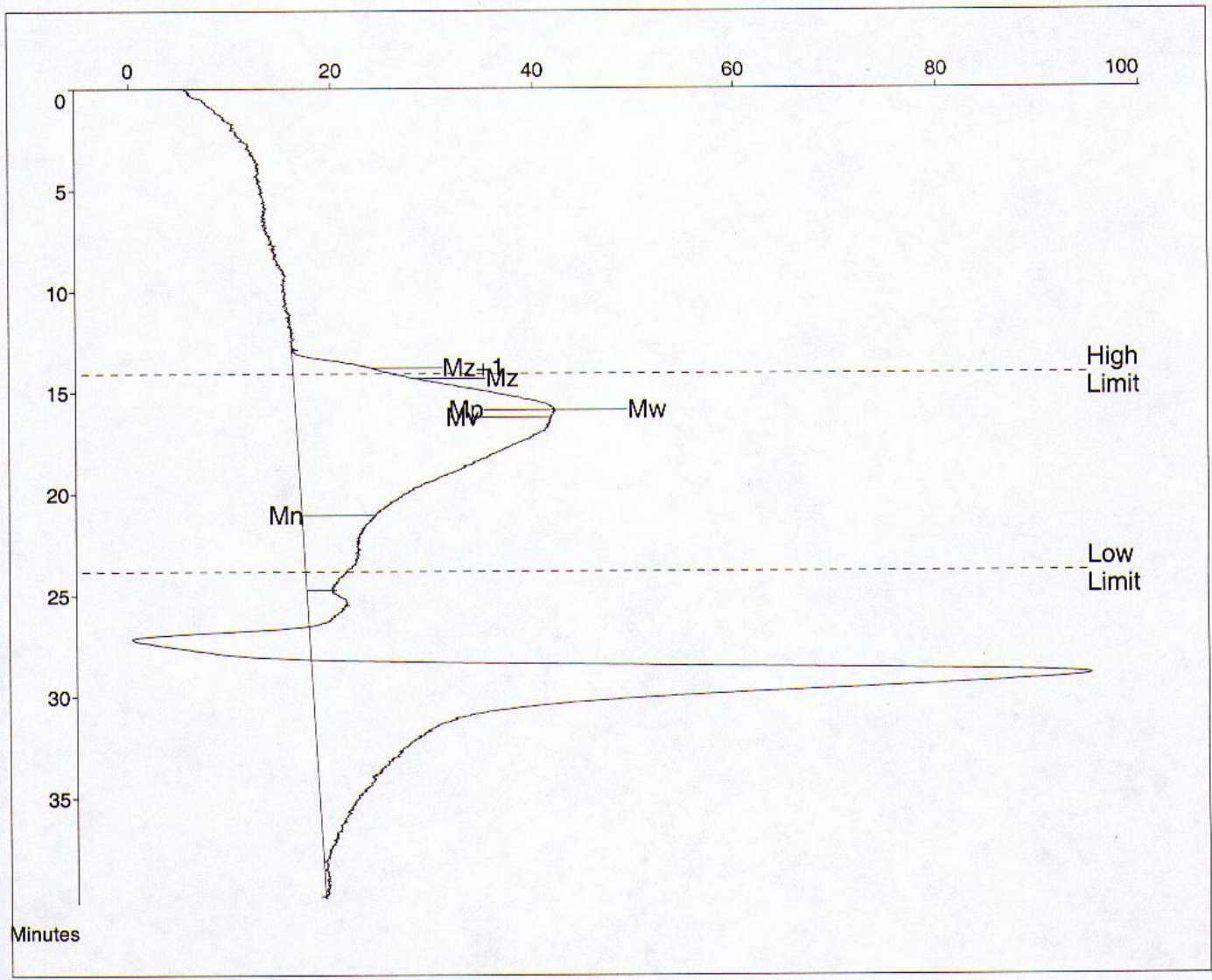

$\begin{array}{lclr}\mathrm{Mp}= & 514856 & \mathrm{Mz}= & 2529239 \\ \mathrm{Mn}= & 4642 & \mathrm{Mz}+1= & 4510765 \\ \mathrm{Mw}= & 513543 & \mathrm{Mv}= & 368945 \\ \text { Polydispersity }= & 110.624 & \text { Peak Area }= & 1803137\end{array}$


V.2. GPC of PE sample, Table 5, entry 2

\section{Polymer Laboratories PL Caliber GPC Software}

\section{Unknown $\quad 85 . \mathrm{HOO}$}

$>P E$

Concentration :

Injection Volume

Solvent

Column Set

Method 1

Comments :

Calibration Using :

Calibration Limits :

Flow Rate Marker :

Narrow Standards

13.95 to 23.63

found at : $\quad 38.08$

Broad Peak Start:

12.77

End: 24.67
09:22 Wed Jun 022004

Acquired : $\quad$ 08:59 Wed Jun 022004

Operator Richard Jordan

Detector :

Temperature :

Flow Rate : 1.000

Standards

Curve Used: $\quad 3$ rd Order Polynomia

Last Calibrated : $\quad$ Wed Jun 02 09:16:52 2004

in Standards at : $\quad 38.42 \quad$ Mins

Mins

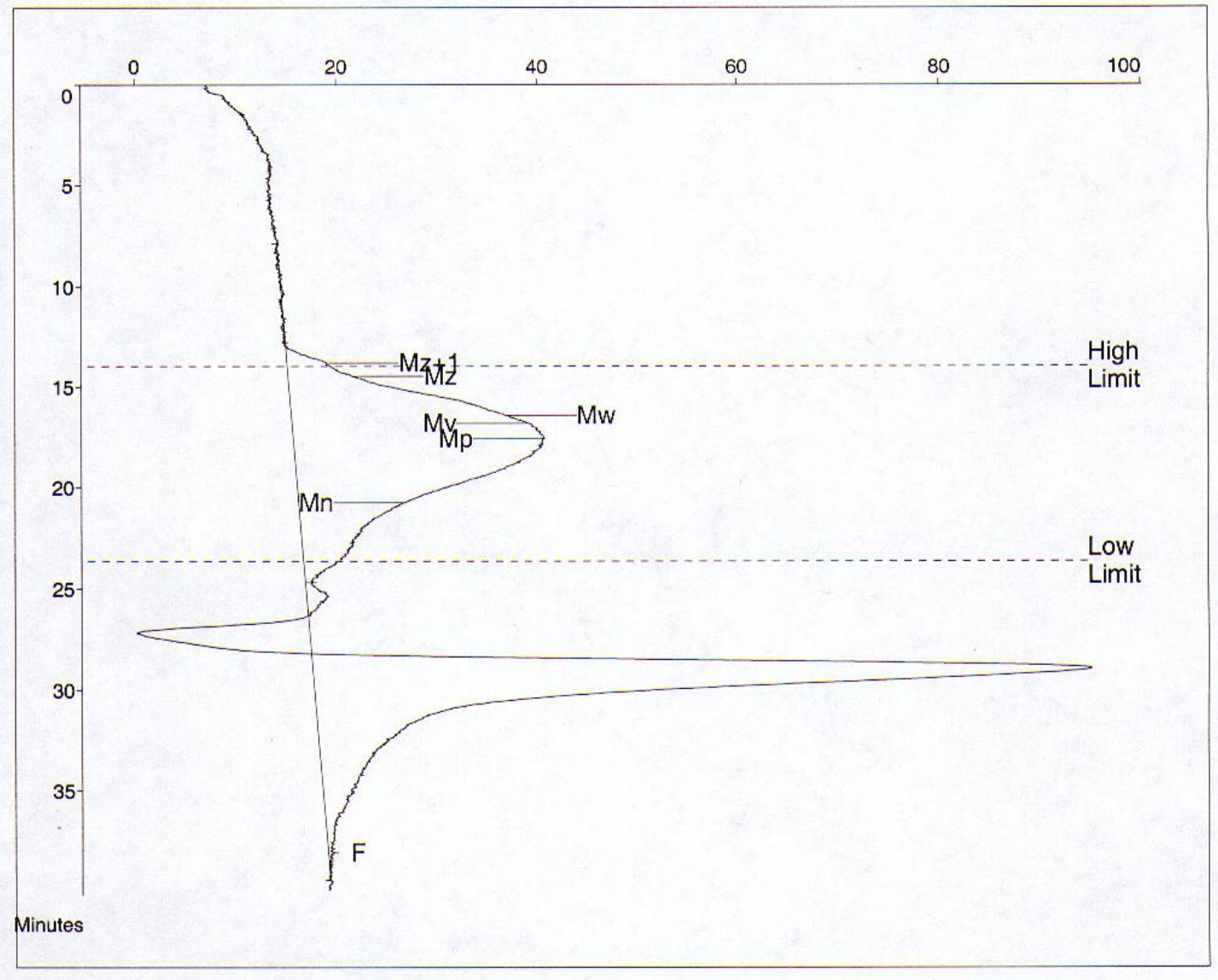

\begin{tabular}{lclc}
\multicolumn{4}{c}{ Molecular Weight Averages } \\
$\mathrm{Mp}=$ & 92911 & $\mathrm{Mz}=$ & 1930429 \\
$\mathrm{Mn}=$ & 5238 & $\mathrm{Mz}+1=$ & 3971747 \\
$\mathrm{Mw}=$ & 274364 & $\mathrm{Mv}=$ & 188674 \\
Polydispersity $=$ & 52.376 & Peak Area $=$ & 1731360
\end{tabular}


V.3. GPC of PE sample, Table 5, entry 8

\section{Polymer Laboratories \\ PL Caliber GPC Software}

\section{Unknown 212.HOO}

$>$ PE

Concentration :

Injection Volume :

Solvent

Column Set :

Method 1

Comments :

Calibration Using :

Calibration Limits :

Narrow Standards

14.08

Flow Rate Marker

Broad Peak Start : to 23.83

to $23.83 \quad$ Mins
found at: Not Found

End: 19.05
09:21 Wed Jun 022004

Acquired : $\quad$ 08:59 Wed Jun 022004

Operator Richard Jordan

Detector

Temperature :

Flow Rate : 1.000

Standards
Urd Order Polynomia

Last Calibrated : $\quad$ Wed Jun 02 09:16:52 2004

in Standards at : $\quad 38.42 \quad$ Mins

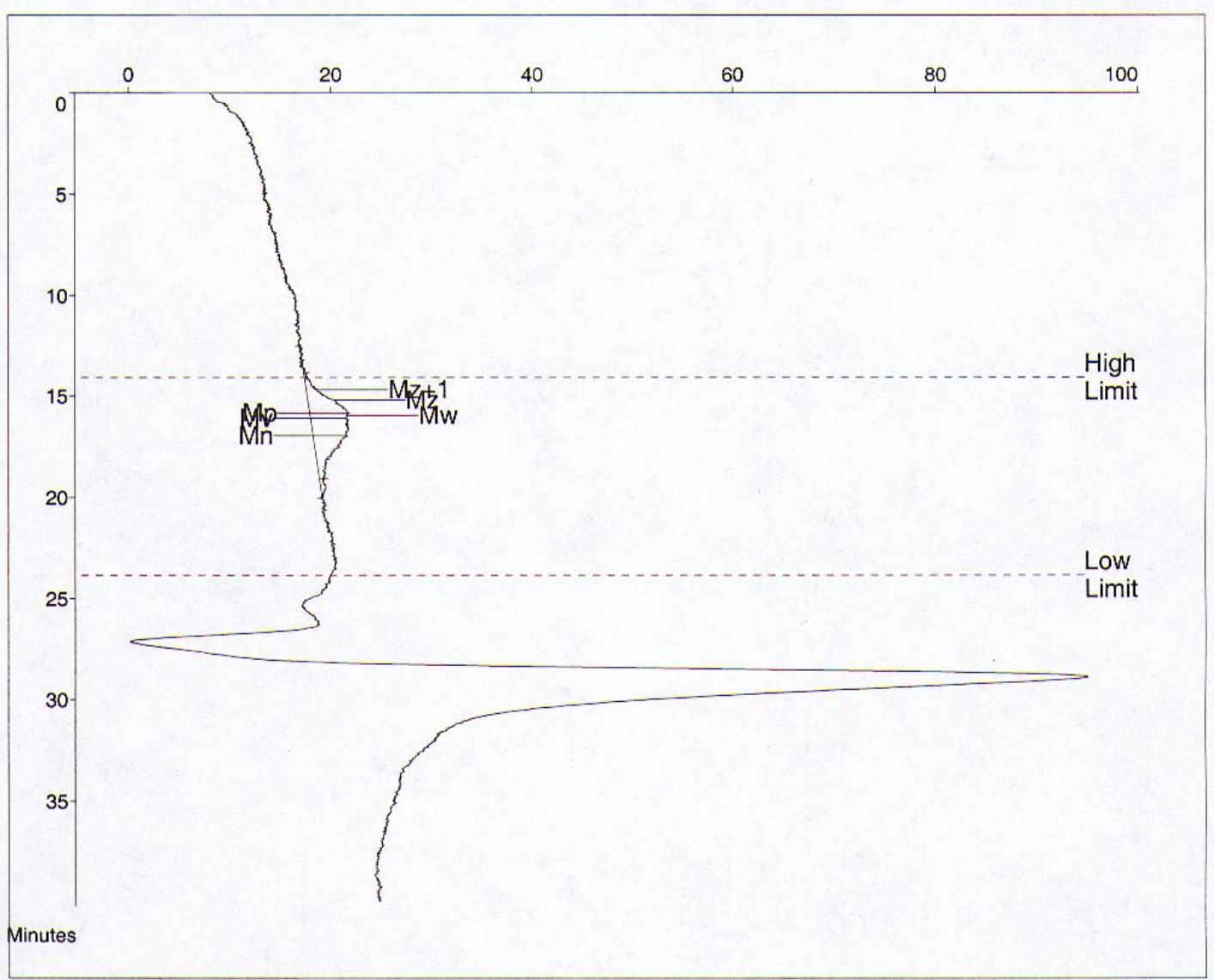

Molecular Weight Averages

$\begin{array}{lrlr}M p= & 540470 & M z= & 1028157 \\ M n= & 188763 & M z+1= & 1727281 \\ M w= & 480268 & M v= & 426320 \\ \text { Polydispersity }= & 2.544 & \text { Peak Area }= & 127793\end{array}$


V.4. DSC of PE sample, Table 5, entry 2

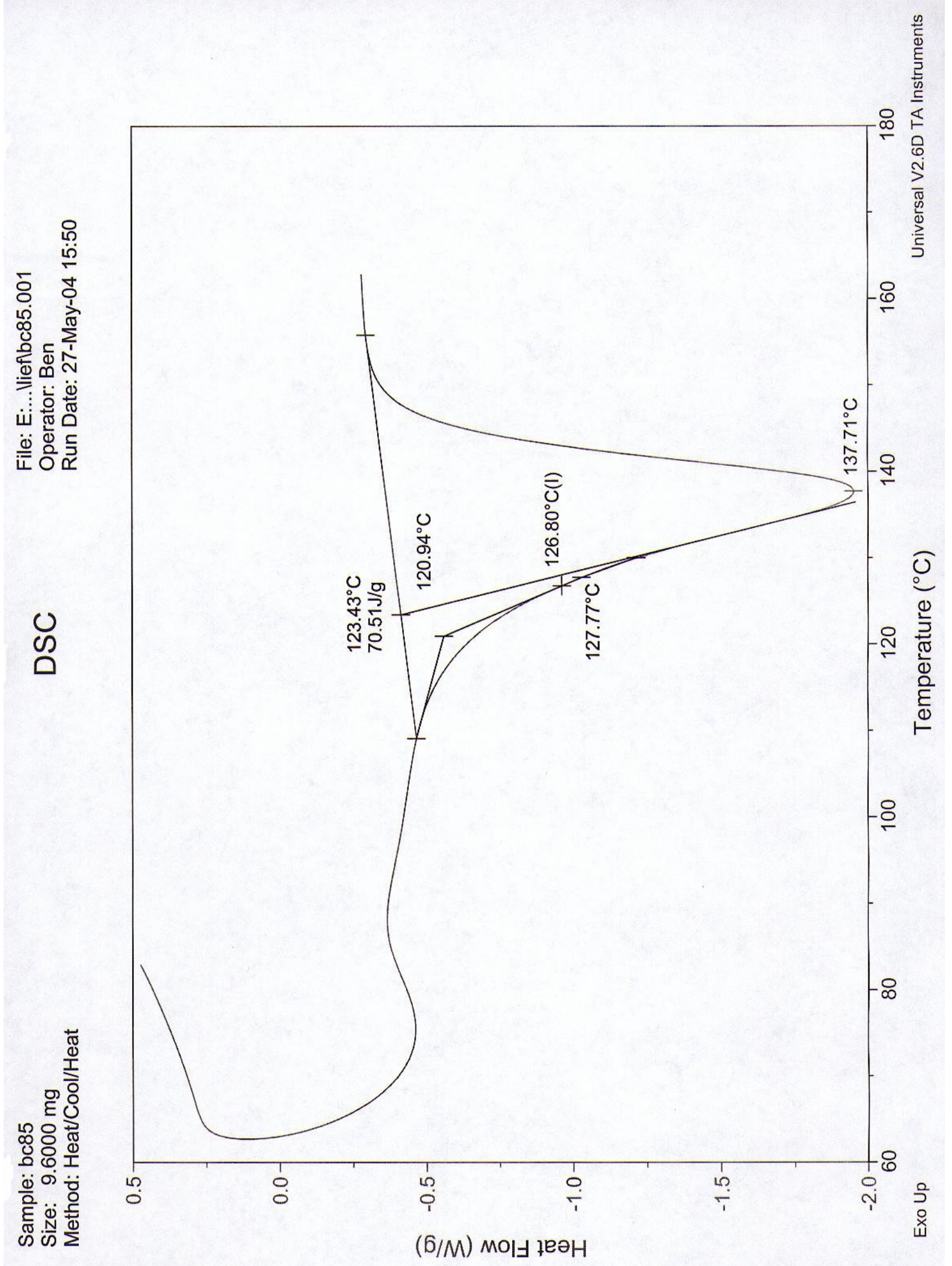


V.5. DSC of PE sample, Table 5, entry 7

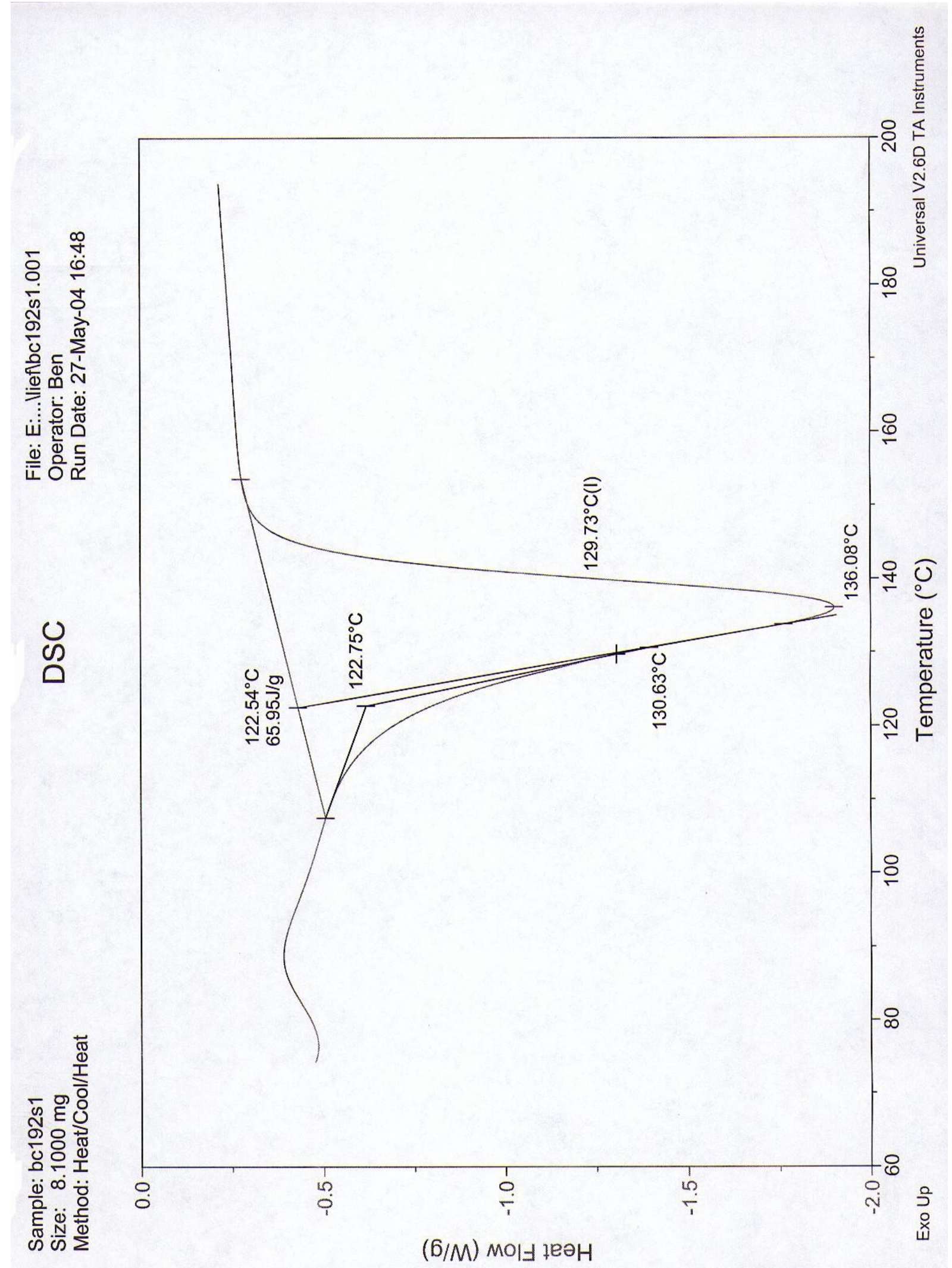

